\title{
Charge stripe order near the surface of 12-percent doped $\mathrm{La}_{2-x} \mathrm{Sr}_{x} \mathrm{CuO}_{4}$
}

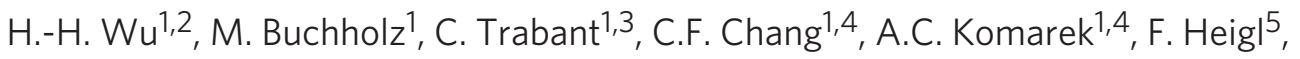 \\ M.v. Zimmermann ${ }^{6}$, M. Cwik ${ }^{1}$, F. Nakamura7 ${ }^{7}$, M. Braden ${ }^{1} \&$ C. Schüßler-Langeheine ${ }^{1,3}$
}

A collective order of spin and charge degrees of freedom into stripes has been predicted to be a possible ground state of hole-doped $\mathrm{CuO}_{2}$ planes, which are the building blocks of hightemperature superconductors. In fact, stripe-like spin and charge order has been observed in various layered cuprate systems. For the prototypical high-temperature superconductor $\mathrm{La}_{2-x} \mathrm{Sr}_{x} \mathrm{CuO}_{4}$, no charge-stripe signal has been found so far, but several indications for a proximity to their formation. Here we report the observation of a pronounced charge-stripe signal in the near surface region of 12 -percent doped $\mathrm{La}_{2}-\mathrm{Sr}_{x} \mathrm{CuO}_{4}$. We conclude that this compound is sufficiently close to charge stripe formation that small perturbations or reduced dimensionality near the surface can stabilize this order. Our finding of different phases in the bulk and near the surface of $\mathrm{La}_{2}-\mathrm{Sr}_{x} \mathrm{CuO}_{4}$ should be relevant for the interpretation of data from surface-sensitive probes, which are widely used for $\mathrm{La}_{2}-x \mathrm{Sr}_{x} \mathrm{CuO}_{4}$ and similar systems.

\footnotetext{
${ }^{1}$ II. Physikalisches Institut, Universität zu Köln, Zülpicher Str. 77, 50937 Köln, Germany. ${ }^{2}$ National Synchrotron Radiation Center, Hsinchu 30076, Taiwan. ${ }^{3}$ Helmholtz-Zentrum Berlin für Materialien und Energie GmbH, Albert-Einstein-St 15, 12489 Berlin, Germany. ${ }^{4}$ Max-Planck Institute CPfS, Nöthnitzer Str. 40, 01187 Dresden, Germany. ${ }^{5}$ ALBA Synchrotron Light Facility, 08290 Cerdanyola del Vallès, Barcelona, Spain. ${ }^{6}$ Hamburger Synchrotronstrahlungslabor HASYLAB at Deutsches Elektronen-Synchrotron DESY, 22603 Hamburg, Germany. ${ }^{7}$ Department of Quantum Matter, ADSM, Hiroshima University, Higashi-Hiroshima 739-8530, Japan. Correspondence and requests for materials should be addressed to C.S.L. (email: christian.schuessler@helmholtz-berlin.de).
} 
$\mathrm{H}$ igh-temperature superconductivity occurs in copper oxide systems that crystallize in a layered perovskite structure. The characteristic building blocks of these systems are well-separated copper-oxide planes (Fig. 1a). In the parent compound of the single-layered cuprate superconductors, $\mathrm{La}_{2} \mathrm{CuO}_{4}$, the $\mathrm{Cu}^{2+}$ ions in these planes carry a spin $(S=1 / 2)$ and couple antiferromagnetically. Chemical doping by replacing trivalent $\mathrm{La}$ with alkaline earth like $\mathrm{Ba}$ or $\mathrm{Sr}$ introduces holes into the system. Doping holes in the $\mathrm{CuO}_{2}$ planes has been suggested to lead to the formation of non-magnetic Zhang-Rice singlets ${ }^{1}$ suppressing the local moment at the individual $\mathrm{Cu}$ sites. As the free mobility of holes in a two-dimensional antiferromagnetic matrix is energetically not favourable, the formation of stripes was postulated ${ }^{2-5}$, in which hole-rich, non-magnetic sites form one-dimensional lines acting as anti-phase domain walls for the antiferromagnetic order on the hole-poor sites (Fig. 1b). Such an arrangement allows holes to hop along the stripes as well as transversal hopping of the whole stripe and avoids magnetic frustration. In the pseudo-gap phases of $\mathrm{HgBa}_{2} \mathrm{CuO}_{4+\delta}(\mathrm{Hg} 1202)^{6,7}, \mathrm{YBa}_{2} \mathrm{Cu}_{3} \mathrm{O}_{6}{ }^{8}$ and under-doped $\mathrm{La}_{2-x} \mathrm{Sr}_{x} \mathrm{CuO}_{4}$ (LSCO) ${ }^{9}$, signatures of another order parameter have been reported with properties as predicted in the orbitalcurrent model ${ }^{10}$. It was proposed that this other order competes with stripe order and that its suppression results in a suppression of high-temperature superconductivity ${ }^{9}$.

Experimentally, stripes were first observed in layered nickelates $^{11,12}$. Stripes in cuprate systems were observed in the lowtemperature tetragonal (LTT) phases of $\mathrm{La}_{2-x-y} \mathrm{Nd}_{y} \mathrm{Sr}_{x} \mathrm{CuO}_{4}$ $(\mathrm{LNSCO})^{13-16}, \mathrm{La}_{2-x} \mathrm{Ba}_{x} \mathrm{CuO}_{4}(\mathrm{LBCO})^{17}, \mathrm{La}_{2-x-y} \mathrm{Eu}_{y} \mathrm{Sr}_{x} \mathrm{CuO}_{4}$ $(\mathrm{LESCO})^{18}$ in neutron and in X-ray diffraction experiments ${ }^{19}$. Recently, stripe order was found in layered cobaltates ${ }^{20}$. In reciprocal space, the charge order $(\mathrm{CO})$ and the spin order $(\mathrm{SO})$ in a stripe phase lead to different, well-separated superstructure reflections: $\mathrm{CO}$ reflections occur in a distance $2 \varepsilon$ around fundamental Bragg peaks, whereas SO reflections are split off by half of that distance, that is, $\varepsilon$, from the $(1 / 2,1 / 2)$ antiferromagnetic positions of the undoped phase (Fig. 1c). The value of $\varepsilon$ depends on the amount of doping ${ }^{21}$. In contrast to a coupled spin-density wave and charge-density wave as found, for example, in chromium metal $^{22}$, the stripe phase is further characterized by a higher transition temperature for $\mathrm{CO}$ than for SO owing to the two-dimensional character of the magnetic order ${ }^{23}$.

For the prototypical high-temperature superconductor LSCO, no charge-stripe signal has been found so far ${ }^{19,24}$. On the other hand, several indications for the proximity to a stripe phase exist: a weak static stripe-like SO signal for doping levels below 0.15 has been observed ${ }^{25,26}$ as well as magnetic correlations indicating fluctuating magnetic stripes with the characteristic relation between doping level and stripe spacing ${ }^{21}$. Although no spatial modulations of the $\mathrm{Cu}-\mathrm{O}$ bond lengths, which are indicative for charge order, were observed for LSCO, the softening and broadening of the corresponding bond-stretching phonon mode was interpreted as a precursor for the formation of charge stripes ${ }^{27}$. The occurrence of static charge-stripe ordering in LNSCO, LBCO and LESCO has been attributed to the LTT symmetry of the respective phases, where tilting around the $\mathrm{Cu}-\mathrm{O}$ bonds generates two distinct in-plane oxygen sites. The LTT tilt pattern is thus likely to stabilize stripe order; it is less clear, however, if it is required. In fact, for $\mathrm{La}_{1.875} \mathrm{Ba}_{0.125-x} \mathrm{Sr}_{x}$ $\mathrm{CuO}_{4}$ stripe-like charge order has been observed in the low-temperature orthorhombic (LTO) phase in vicinity to the low-temperature less orthorhombic (LTLO) phase most likely due to enhanced structural disorder ${ }^{28}$. We show in the following that charge stripes develop in the near-surface region of 12-per cent doped LSCO, but not in the bulk.

\section{Results}

Approach. We studied pieces from the same single crystal of 12-per cent doped LSCO with 2 techniques: resonant soft X-ray diffraction (RSXD), which is moderately surface sensitive with a probing depth of several nanometres, and high-energy X-ray diffraction, which is bulk sensitive with a probing depth of several millimetres. Both techniques are sensitive to charge stripes: RSXD uses the high spectroscopic sensitivity of dipole-allowed excitations into oxygen $2 p$ and transition-metal $3 d$ states to the electronic state of the scattering ions to probe spatial modulations of these states in
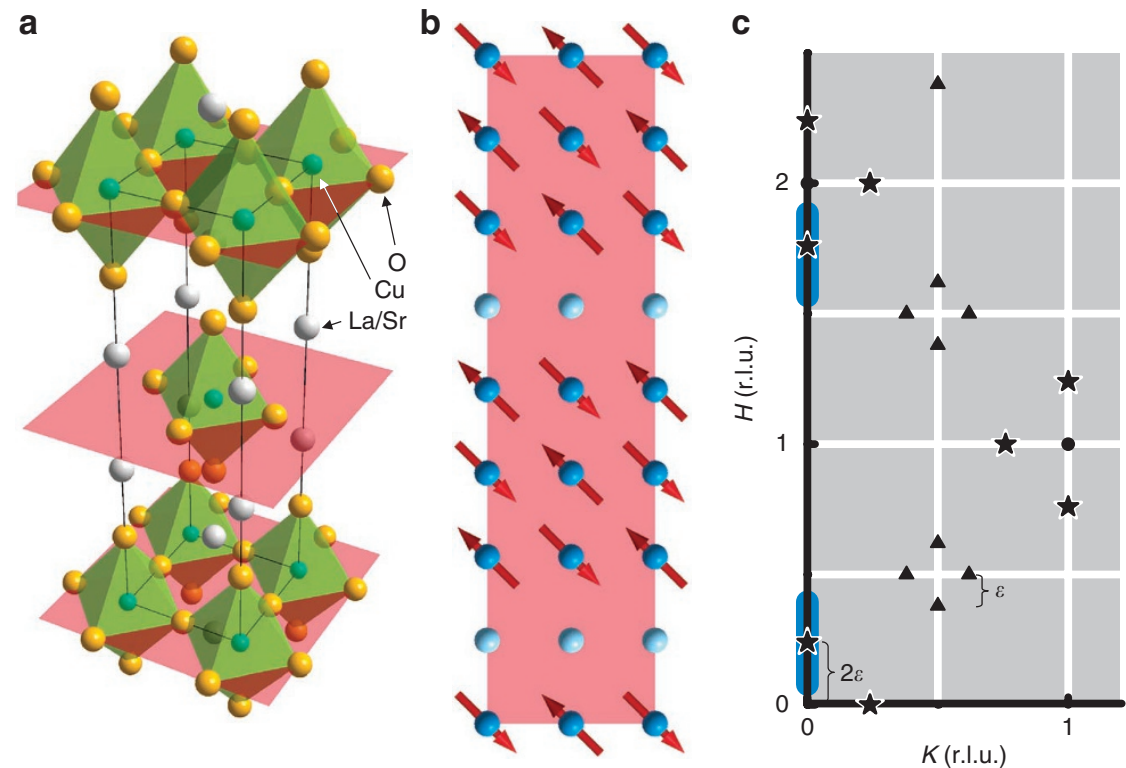

Figure 1 | Stripe order and the observed charge-order peaks. (a) Crystal structure of the layered perovskite cuprates and nickelates. (b) A model picture of site-centred stripe order in the CuO planes (red planes in panel (a)) where only the Cu sites are shown. Light blue denotes hole-rich, dark blue holepoor sites; the arrows indicate the spin direction. (c) Reciprocal space map with fundamental Bragg peaks (filled circles) and spin order (triangles) and charge-order (stars) superstructure reflections related to the stripe phase. The blue lines denote the direction of the scans presented in Fig. 2. 
a diffraction experiment ${ }^{17,18,29,30}$. High-energy X-ray diffraction probes the lattice distortion related to charge order ${ }^{16}$ and allows to detect superstructure reflections that are more than eight orders of magnitude weaker than fundamental Bragg peaks.

Charge stripes near the surface. In the RSXD experiment, we find a clear peak at the CO position (h 00 ), $h \approx 0.24$, at the oxygen
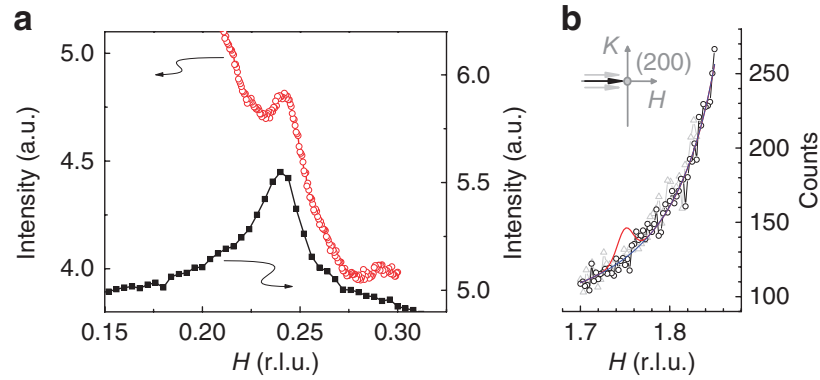

Figure $\mathbf{2}$ | Reciprocal space scans through charge-order positions.

(a) Scans along $[100](H)$ through the $(0.2400)$ superstructure peaks for LSCO (black filled symbols) and LNSCO (red open symbols) recorded at the $\mathrm{O}-\mathrm{K}$ resonance with the samples held at $18 \mathrm{~K}$. The shape of the background is different, but the peaks are clearly visible and of similar intensity. The correlation length along $a$ for LSCO, as determined from the peak width, is about $40 \AA(\approx 10 \mathrm{Cu}$-Cu distances) comparable to that observed for LNSCO $(\approx 50 \AA)$. (b) Example for high-energy X-ray scans through an expected charge-order satellite position near (200) (symbols and blue line) and slightly offset in $K$ (grey symbols) to be sure not to miss any signal. The red line is a simulation of a charge-order peak at what we estimate to be our detection limit, which is of the order of $10^{-8}$ times the intensity of a strong nuclear reflection; note that the width of this simulation corresponds to that seen in the RSXD experiment.
$1 s \rightarrow 2 p$ ( $K$, black curve in Fig. $2 \mathrm{a}$ ) as well as at the copper $2 p \rightarrow 3 d$ $\left(L_{2,3}\right)$ resonances. To identify the electronic character of the peak, we compare its energy dependence with that from an equally doped sample of $\mathrm{La}_{1.48} \mathrm{Nd}_{0.4} \mathrm{Sr}_{0.12} \mathrm{CuO}_{4}$ (LNSCO, red curve in Fig. 2a) for which the existence of static charge stripes has been established by neutron and X-ray diffraction ${ }^{13-16}$. The resonance spectra, that is, the energy dependences of the peak intensity across the resonances are displayed in Fig. 3, together with X-ray absorption spectroscopy data. The resonance spectra are very similar for both systems and resemble those published for charge-order peaks in $\mathrm{LBCO}^{17}$ and in $\mathrm{LESCO}^{18}$, thus proving that charge-stripe order is the common electronic origin of all peaks. The assignment of the LSCO $\left(\begin{array}{lll}0.24 & 0 & 0\end{array}\right)$ peak to charge-stripe order is further confirmed by its temperature dependence. The decay of the LSCO (0.24 00 )-peak intensity on heating is displayed in Fig. $4 \mathrm{a}$. The peak vanishes around $55 \mathrm{~K}$. For comparison, the susceptibility is plotted (Fig. 4b), which shows a critical temperature for superconductivity at $T_{c}=30 \mathrm{~K}$. From neutron scattering experiments using a sample with very similar composition, it is known that the very weak magnetic ordering melts at about the same temperature as superconductivity ${ }^{25}$, which is much lower than what we find for the disappearance of the $(0.2400)$ peak. This behaviour with $T_{\mathrm{CO}}>T_{\mathrm{N}}$ is exactly what is expected for stripe order in layered cuprates ${ }^{23}$.

No charge stripes in the bulk. Our RSXD experiment shows clearly the existence of charge-stripe order in 12-per cent doped LSCO. On the other hand, in our high-energy X-ray diffraction data, no trace of charge order can be found. We scanned at various possible charge-order positions without any positive results. An example scan is presented in Fig. 2b. Combining these two results, we conclude that charge-stripe order does exist in the near-surface region of LSCO but not in the bulk. From the RSXD peak profile, we determine the coherence length of charge order perpendicular to the (100)-oriented surface to be at least in the range between 4 and $6 \mathrm{~nm}$. This is shorter than the probing depth at the oxygen- $K$
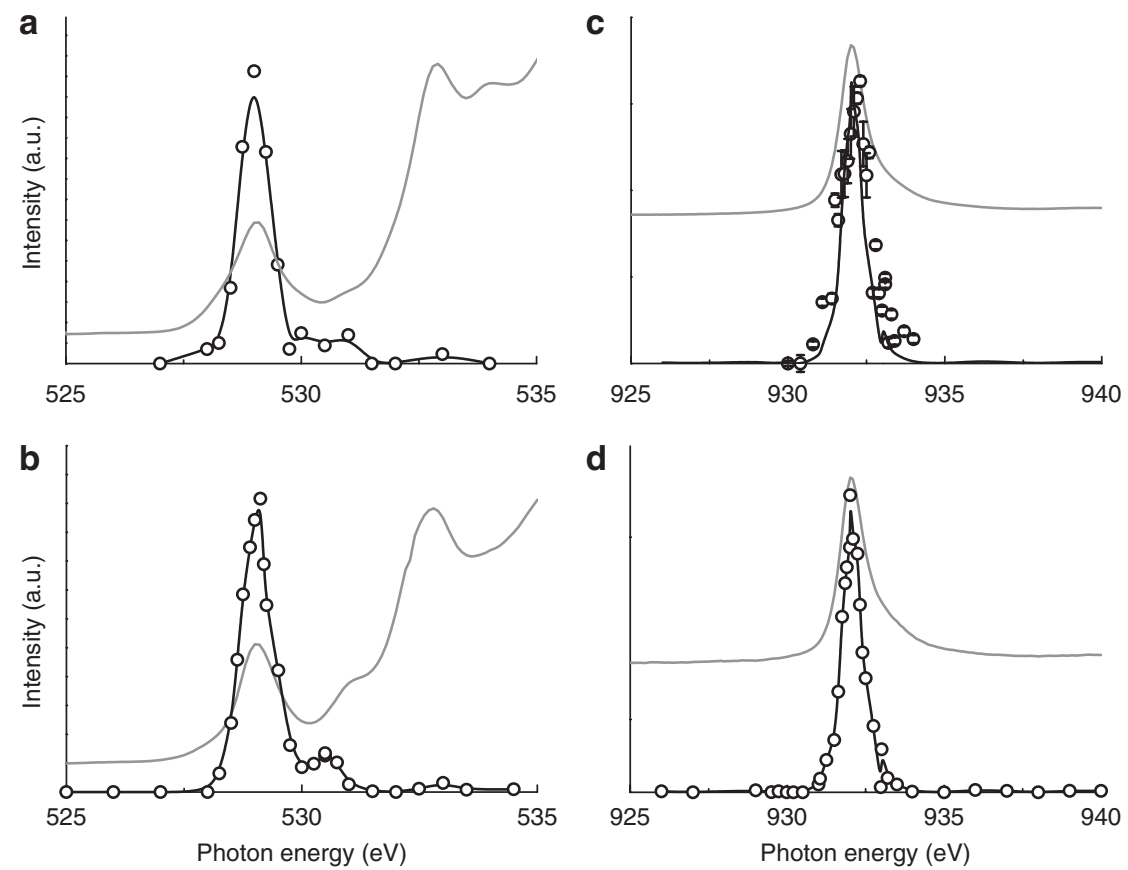

Figure $\mathbf{3}$ | Resonance spectra from LSCO and LNSCO. Energy dependence of the (0.24 0 0) RSXD signals (symbols) as observed at the oxygen $1 s \rightarrow 2 p(\mathrm{O}-K)$ resonance for LSCO (a) and LNSCO (b) and at the copper $2 p_{3 / 2} \rightarrow 3 d\left(L_{3}\right)$ resonance for LSCO (c) and LNSCO (d). The black lines through the symbols are guides to the eye; for comparison, these lines are identical in (c) and (d). The respective X-ray absorption signals (grey line) are shown as energy references. 

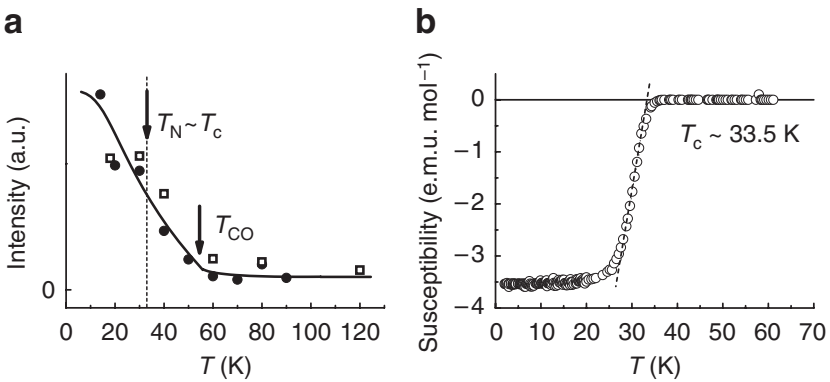

Figure 4 | Temperature dependence. (a) Temperature dependence of the $(0.2400)$ RSXD signal in LSCO as recorded at the $\mathrm{Cu}-\mathrm{L}_{3}$ (filled symbols) and $\mathrm{O}-\mathrm{K}$ resonance (open symbols). (b) Susceptibility, which indicates superconductivity below $30 \mathrm{~K}$. The (0.24 00 ) signal is observable up to about $55 \mathrm{~K}$ ruling out a magnetic origin of this peak.

resonance and hence gives a lower limit for the thickness of the charge-ordered surface region. The sensitivity of the high-energy $\mathrm{X}$-ray diffraction experiment was high enough to exclude a possible scenario of such short-range-correlated charge order in the whole sample volume. We only find it near the surface.

Surface-preparation effects. To clarify whether strain caused by polishing induced charge order, we carried our RSXD experiments from in-situ cleaved samples. For these a (001) surface orientation is determined by the cleavage plane of LSCO and $(0.2400)$ is not accessible. From other cuprates, it is known that the charge-order peak is visible over a wide range of L-values ${ }^{16}$ and indeed, we found a charge-order signal at $(0.240 \ell)$ with $0.65 \leq \ell \leq 0.8$, which exhibits the same dependence on temperature and energy as the $\left(\begin{array}{lll}0.24 & 0 & 0\end{array}\right)$ peak from the (100)-oriented samples. The occurrence of charge order in LSCO is hence neither a property of a particular surface nor is it induced by a particular surface preparation method. It is an intrinsic property of the near-surface region, where reduced dimensionality or structural relaxations apparently stabilize an order, which does not form in the bulk. We note that we did not find any signal from charge stripes in a surface-sensitive RSXD experiment from a 14-per cent doped LSCO sample.

\section{Discussion}

The observation of stabilized charge order in the near-surface region of 12-per cent doped LSCO is surprising, as one usually expects a three-dimensional crystal to start melting at the surface. We tentatively attribute the enhanced stability of the charge ordering at the surface to the documented impact of disorder on local tilting and on electronic mobility. The structural disorder should be larger at the surface and it induces charge ordering in the $\mathrm{La}_{1.875} \mathrm{Ba}_{0.125-x}$ $\mathrm{Sr}_{x} \mathrm{CuO}_{4}$ series. In nickelates ${ }^{31}$ and in manganates ${ }^{32}$, also, disorder stabilizes stripe or other types of charge-ordered phases.

The fact that the near-surface region of LSCO is different from the bulk should be considered when surface-sensitive experiments from LSCO and related systems are interpreted in terms of bulk properties of the material. Stripes are known to affect the shape of the Fermi surface as seen in angle-resolved photoemission experiments ${ }^{33-36}$. Our finding may, for example, help to relate the apparent similarities in the Fermi-surfaces of $\mathrm{LNSCO}^{34}$ and $\mathrm{LSCO}^{35}$ to the different superconducting properties of these compounds. In scanning tunnelling spectroscopy experiments, spatial modulations of the local density of states have been observed for different cuprate systems ${ }^{37,38}$. For 12-per cent doped LSCO spatial modulations on different length scales have been observed ${ }^{39}$; for the interpretation of these, the existence of charge stripes may matter ${ }^{40}$.

In conclusion, RSXD experiments prove the existence of charge-stripe order in an at least 4-nm thick, near-surface region of 12-per cent doped LSCO, while at the same time, there is no indication for such kind of order in the bulk. The near-surface order does not depend on the surface orientation and preparation method, but seems to be an intrinsic property of the surface. The well-established proximity of LSCO to the formation of charge stripes is apparently sufficient to drive the phase transition near the surface.

\section{Methods}

Samples. Single crystals of $\mathrm{La}_{1.88} \mathrm{Sr}_{0.12} \mathrm{CuO}_{4}$ and $\mathrm{La}_{1.48} \mathrm{Nd}_{0.4} \mathrm{Sr}_{0.12} \mathrm{CuO}_{4}$ were prepared by floating-zone method using an image furnace. The indexation of reciprocal space points refers to the high-temperature tetragonal unit cell with $a \approx 3.78 \AA$ and $c \approx 13.2 \AA$.

Resonant soft X-ray diffraction and X-ray absorption. The RSXD measurements were carried out at UE46-PGM-1 beamline at the synchrotron-radiation source BESSY II operated by the Helmholtz-Zentrum Berlin. The two-circle ultrahigh vacuum diffractometer designed at Freie Universität Berlin was used in horizontal scattering geometry. The polarization vector of the incoming X-rays was perpendicular to the scattering plane, and parallel to the $b$ axis of the single crystals. For the RSXD experiment, two different sample orientations were used: samples with a (100) surface normal were polished ex situ; those with a (001) surface normal were cleaved in situ. The energy dependence of the diffraction features was determined by taking scans along [100] through the peak (similar to Fig. 2a) for every photon energy. The X-ray absorption spectroscopy spectra were obtained from the total-electron yield signal recorded as the sample drain current. The energy resolution of X-rays near the oxygen $K$ edge and the copper $L$ edge are $\sim 90$ and $210 \mathrm{meV}$, respectively.

High-energy X-ray diffraction and susceptibility. High-energy X-ray diffraction experiments were carried out at beamline BW5 at DESY using $100 \mathrm{keV}$ X-rays. Susceptibility measurements were carried out in a field of 50 Oe after zero-field cooling using a small piece of the same single crystal as used for the diffraction experiments.

\section{References}

1. Zhang, F. C. \& Rice, T. M. Effective Hamiltonian for the superconducting Cu oxides. Phys. Rev. B 37, 3759-3761 (1988).

2. Zaanen, J. \& Gunnarsson, O. Charged magnetic domain lines and the magnetism of high- $T_{c}$ oxides. Phys. Rev. B 40, 7391-7394 (1989).

3. Emery, V. J., Kivelson, S. A. \& Zachar, O. Spin-gap proximity effect mechanism of high-temperature superconductivity. Phys. Rev. B 56, 6120-6147 (1997).

4. Emery, V. J., Kivelson, S. A. \& Tranquada, J. M. Stripe phases in hightemperature superconductors. Proc. Natl Acad. Sci. USA 96, 8814-8817 (1999).

5. Wilson, J. A. Structural matters in HTSC: the origin and form of stripe organization and checkerboarding. J. Phys. 18, R69-R99 (2006).

6. Li, Y. et al. Unusual magnetic order in the pseudogap region of the superconductor $\mathrm{HgBa}_{2} \mathrm{CuO}_{4+\delta}$. Nature 455, 372-375 (2008).

7. Li, Y. et al. Hidden magnetic excitation in the pseudogap phase of a high- $T$ superconductor. Nature 468, 283-285 (2010).

8. Fauqué, B. et al. Magnetic order in the pseudogap phase of high-TC superconductors. Phys. Rev. Lett. 96, 197001 (2006).

9. Balédent, V. et al. Two-dimensional orbital-like magnetic order in the hightemperature $\mathrm{La}_{2-x} \mathrm{Sr}_{x} \mathrm{CuO}_{4}$ superconductor. Phys. Rev. Lett. 105, 027004 (2010)

10. Varma, C. M. Theory of the pseudogap state of the cuprates. Phys. Rev. B 73, 155113 (2006).

11. Chen, C. H., Cheong, S. W. \& Cooper, A. S. Charge modulations in $\mathrm{La}_{2}{ }_{-} \mathrm{Sr}_{x} \mathrm{NiO}_{4+y}$ : ordering of polarons. Phys. Rev. Lett. 71, 2461-2464 (1993)

12. Tranquada, J. M., Buttrey, D. J. \& Rice, D. E. Phase separation, charge-density waves, and magnetism in $\mathrm{La}_{2} \mathrm{NiO}_{4+\delta}$ with $\delta=0.105$. Phys. Rev. Lett. 70, 445-448 (1993).

13. Tranquada, J. M., Sternlieb, B. J., Axe, J. D., Nakamura, Y. \& Uchida, S. Evidence for stripe correlations of spins and holes in copper oxide superconductors. Nature 375, 561-563 (1995).

14. Tranquada, J. M. et al. Neutron-scattering study of stripe-phase order of holes and spins in $\mathrm{La}_{1.48} \mathrm{Nd}_{0.4} \mathrm{Sr}_{0.12} \mathrm{CuO}_{4}$. Phys. Rev. B 54, 7489-7499 (1996)

15. Tranquada, J. M. et al. Coexistence of, and competition between, superconductivity and charge-stripe order in $\mathrm{La}_{1.6-x} \mathrm{Nd}_{0.4} \mathrm{Sr} x \mathrm{CuO}_{4}$. Phys. Rev. Lett. 78, 338-341 (1997).

16. Zimmermann, M. V. et al. Hard-X-ray diffraction study of charge stripe order in $\mathrm{La}_{1.48} \mathrm{Nd}_{0.4} \mathrm{Sr}_{0.12} \mathrm{CuO}_{4}$. Europhys. Lett. 41, 629-634 (1998).

17. Abbamonte, P. et al. Spatially modulated 'Mottness' in $\mathrm{La}_{2-x} \mathrm{Ba}_{x} \mathrm{CuO}_{4}$. Nat. Phys. 1, 155-158 (2005).

18. Fink, J. et al. Charge ordering in $\mathrm{La}_{1,8-} \mathrm{Eu}_{0,2} \mathrm{Sr}_{x} \mathrm{CuO}_{4}$ studied by resonant soft x-ray diffraction. Phys. Rev. B 79, 100502 (2009).

19. Vojta, M. Lattice symmetry breaking in cuprate superconductors: stripes, nematics, and superconductivity. Adv. Phys. 58, 699-820 (2009). 
20. Cwik, M. et al. Magnetic correlations in $\mathrm{La}_{2}-x \mathrm{Sr}_{x} \mathrm{CoO}_{4}$ studied by neutron scattering: possible evidence for stripe phases. Phys. Rev. Lett. 102, 057201 (2009).

21. Yamada, K. et al. Doping dependence of the spatially modulated dynamical spin correlations and the superconducting-transition temperature in $\mathrm{La}_{2-x} \mathrm{Sr}_{x} \mathrm{CuO}_{4}$. Phys. Rev. B 57, 6165-6172 (1998).

22. Pynn, R., Press, W., Shapiro, S. M. \& Werner, S. A. Second and third harmonics of the spin density wave in chromium metal. Phys. Rev. B 13, 295-298 (1976).

23. Zachar, O., Kivelson, S. A. \& Emery, V. J. Landau theory of stripe phases in cuprates and nickelates. Phys. Rev. B 57, 1422-1426 (1998).

24. Fujita, M., Goka, H., Yamada, K. \& Matsuda, M. Competition between chargeand spin-density-wave order and superconductivity in $\mathrm{La}_{1.875} \mathrm{Ba}_{0.125-x} \mathrm{Sr}_{x} \mathrm{CuO}_{4}$. Phys. Rev. Lett. 88, 167008 (2002).

25. Kimura, H. et al. Neutron-scattering study of static antiferromagnetic correlations in $\mathrm{La}_{2-x} \mathrm{Sr}_{x} \mathrm{Cu}_{1-y} \mathrm{Zn}_{y} \mathrm{O}_{4}$. Phys. Rev. B 59, 6517-6523 (1999).

26. Suzuki, T. et al. Observation of modulated magnetic long-range order in $\mathrm{La}_{1.88} \mathrm{Sr}_{0.12} \mathrm{CuO}_{4}$. Phys. Rev. B 57, R3229-R3232 (1998).

27. Pintschovius, L. \& Braden, M. Anomalous dispersion of LO phonons in $\mathrm{La}_{1.85} \mathrm{Sr}_{0.15} \mathrm{CuO}_{4}$. Phys. Rev. B 60, R15039-R15042 (1999).

28. Kimura, H. et al. Relationship between charge stripe order and structural phase transitions in $\mathrm{La}_{1.875} \mathrm{Ba}_{0.125-x} \mathrm{Sr}_{x} \mathrm{CuO}_{4}$. Phys. Rev. B 70, 134512 (2004).

29. Abbamonte, P. et al. Structural probe of the doped holes in cuprate superconductors. Science 297, 581-584 (2002).

30. Schüßler-Langeheine, C. et al. Spectroscopy of stripe order in $\mathrm{La}_{1.8} \mathrm{Sr}_{0.2} \mathrm{NiO}_{4}$ using resonant soft x-ray diffraction. Phys. Rev. Lett. 95, 156402 (2005).

31. Yu, X. Z. et al. Effect of quenched disorder on charge ordering structure in $R E_{1.67} A E_{0.33} \mathrm{NiO}_{4}$ (RE=La, Pr, Nd, Sm; $\left.A E=\mathrm{Ca}, \mathrm{Sr}\right)$. J. Phys. Soc. Jpn 77, 093709 (2008).

32. Tokura, Y. \& Tomioka, Y. Colossal magnetoresistive manganites. J. Magn. Magn. Mater. 200, 1-23 (1999).

33. Damascelli, A., Hussain, Z. \& Shen, Z.- X. Angle-resolved photoemission studies of the cuprate superconductors. Rev. Mod. Phys. 75, 473-541 (2003).

34. Zhou, X. J. et al. One-dimensional electronic structure and suppression of $d$-wave node state in $\left(\mathrm{La}_{1.28} \mathrm{Nd}_{0.6} \mathrm{Sr}_{0.12}\right) \mathrm{CuO}_{4}$. Science 286, 268-272 (1999).

35. Ino, A. et al. Electronic structure of $\mathrm{La}_{2}{ }_{-} \mathrm{Sr}_{x} \mathrm{CuO}_{4}$ in the vicinity of the superconductor-insulator transition. Phys. Rev. B 62, 4137-4141 (2000).
36. Hong-Min, J., Cui-Ping, C. \& Jian-Xin, L. Spectral and optical properties in the antiphase stripe phase of the cuprate superconductors. J. Phys. 21, 375701 (2009).

37. Hanaguri, T. et al. A 'checkerboard' electronic crystal state in lightly hole-doped $\mathrm{Ca}_{2}{ }_{x} \mathrm{Na}_{x} \mathrm{CuO}_{2} \mathrm{Cl}_{2}$. Nature 430, 1001-1005 (2004).

38. Bonnell, D. A. \& Garra, J. Scanning probe microscopy of oxide surfaces: atomic structure and properties. Rep. Prog. Phys. 71, 044501 (2008).

39. Yuli, O., Asulin, I., Koren, G. \& Millo, O. Spatial modulation of midgap states in (001) $\mathrm{La}_{1.88} \mathrm{Sr}_{0.12} \mathrm{CuO}_{4}$ films: Indications for antiphase ordering of the $d$-wave order parameter. Phys. Rev. B 81, 024516 (2010).

40. Kai-Yu, Y., Wei Qiang, C., Rice, T. M., Sigrist, M. \& Fu-Chun, Z. Nature of stripes in the generalized $t-J$ model applied to the cuprate superconductors. New J. Phys 11, 055053 (2009).

\section{Acknowledgements}

We thank E. Schierle for expert experimental support and helpful discussions. Experimental support by D. Schmitz, L. Hamdan and S. Heijligen is gratefully acknowledged. Work in Cologne was funded by the DFG through SFB 608 and by the BMBF through projects $05 \mathrm{KS} 7 \mathrm{PK} 1$ and $05 \mathrm{~K} 10 \mathrm{PK} 2$; the experiments by BESSY were financially supported by the BMBF (05ES3XBA/5) and by the Helmholtz-Zentrum Berlin.

\section{Author contributions}

M.Br. and C.S.-L. planned the experiment. H.-H.W., M.Bu., C.T., C.F.C., F.H., M.C. and C.S.-L. carried out the soft X-ray diffraction experiment and analysed the data. A.C.K. and M.v.Z. carried out the high-energy diffraction experiment and analysed the data. F.N. grew the samples. H.-H.W., M.Br. and C.S.-L. wrote the manuscript with contributions from the other authors.

\section{Additional information}

Competing financial interests: The authors declare no competing financial interests.

Reprints and permission information is available online at http://npg.nature.com/ reprintsandpermissions/

How to cite this article: $\mathrm{Wu}, \mathrm{H} .-\mathrm{H}$. et al. Charge stripe order near the surface of 12-per cent doped $\mathrm{La}_{2-x} \mathrm{Sr}_{x} \mathrm{CuO}_{4}$. Nat. Commun. 3:1023 doi: 10.1038/ncomms2019 (2012). 